\title{
VSMC MIMO: A Spectral Efficient Scheme for Cooperative Relay in Cognitive Radio Networks
}

\author{
Chao Kong*, Zengwen Yuan*, Xushen Han*, Feng Yang*, Xinbing Wang ${ }^{\dagger \ddagger}$, Tao Wang ${ }^{\S}$, Songwu Lu \\ ${ }^{*}$ Department of Electronic Engineering, Shanghai Jiao Tong University, China \\ ${ }^{\dagger}$ School of Electronic, Info. \& Electrical Engineering, Shanghai Jiao Tong University, China \\ ${ }^{\ddagger}$ National Mobile Communications Research Laboratory, Southeast University, China \\ ${ }^{\S}$ School of EECS, Peking University, China \\ IDepartment of Computer Science, University of California, Los Angeles, USA \\ Email: \{kongchao, dayflower, edixius, yangfeng, xwang8\}@sjtu.edu.cn,wangtao@pku.edu.cn, slu@cs.ucla.edu
}

\begin{abstract}
Multiple-Input Multiple-Output (MIMO) technology has become an efficient way to improve the capacity and reliability of wireless networks. Traditional MIMO schemes are designed mainly for the scenario of contiguous spectrum ranges. However, in cognitive radio networks, the available spectrum is discontiguous, making traditional MIMO schemes inefficient for spectrum usage. This motivates the design of new MIMO schemes that apply to networks with discontiguous spectrum ranges. In this paper, we propose a scheme called VSMC MIMO, which enables MIMO nodes to transmit variable numbers of streams in multiple discontinuous spectrum ranges. This scheme can largely improve the spectrum utilization and meanwhile maintain the same spatial multiplexing and diversity gains as traditional MIMO schemes. To implement this spectral-efficient scheme on cooperative MIMO relays in cognitive radio networks, we propose a joint relay selection and spectrum allocation algorithm and a corresponding MAC protocol for the system. We also build a testbed by the Universal Software Radio Peripherals (USRPs) to evaluate the performances of the proposed scheme in practical networks. The experimental results show that VSMC MIMO can efficiently utilize the discontiguous spectrum and greatly improve the throughput of cognitive radio networks.
\end{abstract}

\section{INTRODUCTION}

MIMO technology has become an efficient way to improve spectrum efficiency, and is adopted in WLAN, WiMAX and LTE. Multi-user MIMO system can be deployed in cognitive radio (CR) [1][2][3][4] networks. Traditional MIMO schemes are designed mainly for the scenario of contiguous spectrum ranges. However, in $\mathrm{CR}$ networks, the available spectrum is discontiguous, and they can be combined into multiple nonadjacent channels. To make full use of spectrum resources in CR networks, a question that how MIMO nodes operate on multiple nonadjacent channels raises.

In this paper, we propose a new scheme: VSMC MIMO (Variable numbers of Streams on Multiple Channels of MIMO system). The VSMC MIMO scheme allows MIMO nodes to transmit or receive variable numbers of data streams on multiple channels, thus this scheme can significantly improve the throughput or reliability of data transmission and make full use of spectrum resources.

We give a simple example to illustrate the VSMC MIMO scheme. As shown in Fig. 1, there are one transmitter $S$ and three receivers $R 1, R 2$, and $R 3$. The numbers of antennas

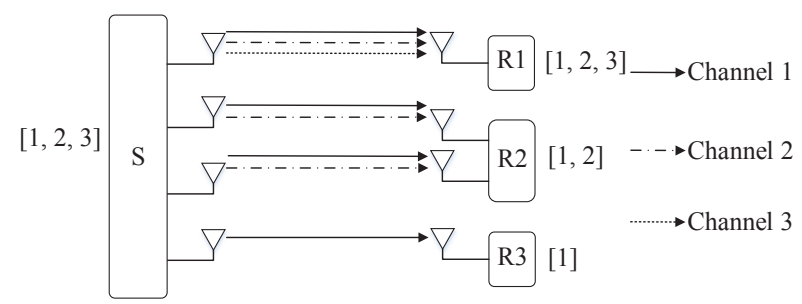

Fig. 1. VSMC scheme: variable numbers of streams on multiple channels The arrow lines indicate the numbers of data streams between transmitters and receivers, and do not mean the actual signals at the antennas. All the figures in this paper adopt this approach for simpler illustration.

of them are four, one, two and one. We regard the four nodes as a "virtual MIMO" system. With techniques such as beamforming, $S$ can transmit four streams concurrently to $R 1$, $R 2$ and $R 3$. Since that the spectrum are occupied by primary users (PUs), the available channels at four nodes are different, as shown in Fig. 1. Nodes can communicate with each other if they share common channels. In the traditional approach, we can transmit four streams concurrently on channel one. This approach wastes the available channel two and channel three. In contrast, by using our VSMC MIMO scheme, $S$ can transmit four streams to $R 1, R 2$ and $R 3$ on channel one, three streams to $R 1$ and $R 2$ on channel two and one stream to $R 1$ on channel three concurrently.

There are several challenges to realize the VSMC MIMO scheme:

- Traditionally, one radio only operates on one channel. But in VSMC MIMO scheme, one radio should transmit or receive signals on multiple (probably discontiguous) channels simultaneously. Also, inter-channel interferences should be eliminated to ensure correct transmission.

- In most MU-MIMO systems, MIMO nodes only transmit message in a contiguous frequency range, so the Channel State Information (CSI) is easy to estimate. However, in VSMC MIMO scheme, MIMO nodes have to calculate C$\mathrm{SI}$ of several discontiguous frequency ranges. In addition, the VSMC MIMO scheme allows variable numbers of streams transmitted on multiple channels, so data streams on different channels should be processed individually, which complicates the signal processing. 
In cognitive radio networks, the available spectrum and traffic demands of MIMO nodes may be different. Some MIMO nodes have low traffic demands and abundant spectrum resources, while other MIMO nodes have poor resources and they can not meet their traffic demands, so resource abundant MIMO nodes can act as relays to leverage traffic demands of resource poor MIMO nodes [5][6]. The VSMC MIMO scheme is very suitable for implementing MIMO relays in cognitive radio networks since this scheme can effectively use the spectrum resources.

In our paper, we design the VSMC MIMO scheme to deal with spectrum heterogeneity at MIMO nodes and implement a real system of cooperative MIMO relay that involves VSMC MIMO scheme based on USRP platform. The contributions of this work are as follows:

- To our best knowledge, this work is the first one to design a feasible and efficient scheme for MIMO nodes to transmit variable numbers of streams on multiple channels. The proposed VSMC MIMO scheme can make full use of available spectrum and achieve spatial multiplexing/diversity gain of MIMO system. In addition, we use the VSMC MIMO scheme to implement MIMO relays in cognitive radio networks.

- We define the relay selection and channel allocation problem to implement cooperative MIMO relay in cognitive radio networks, and propose an algorithm to address it. To make the MIMO relay scheme workable and reliable, we also propose a MAC layer protocol to deal with the coordination and synchronization of all the nodes in cognitive radio networks.

- We build a system of cooperative MIMO relay that involves VSMC MIMO scheme based on USRP platform. The experimental results show that implementing MIMO relays with VSMC MIMO scheme can significantly improve the total throughput of the network.

The organization of the rest of this paper is as follows: Section II introduces related work. We describe how to use VSMC MIMO scheme to implement cooperative MIMO relays in Section III. The relay selection and channel allocation problem for implementing cooperative MIMO relay in cognitive radio networks is solved in Section IV. A MAC layer protocol is proposed to coordinate all the nodes in cognitive radio networks in Section V. In Section VI, we describe the system of cooperative MIMO relays in cognitive radio networks, and evaluate the experimental results. Conclusion is presented in Section VII.

\section{RELATED WORK}

There are many studies on cooperative relay and resource allocation in cognitive radio networks [7][8][9]. Jia et al. first proposed a cooperative relay scheme in cognitive radio networks and implemented a prototype [7]. They improved the prototype and comprehensively analyzed the performance of cooperative relay implementation [8]. In [9], Jia et al. investigated the throughput and delay scaling law in the heterogeneous cognitive radio networks with cooperative secondary users. However, these works only considered SISO nodes in cognitive radio networks. Deploying MIMO nodes in cognitive radio networks was discussed in [10][11][12][13][14][15] [16]. In [10][11], the authors mainly focused on physical layer improvements of MIMO relay. Qin et al. in [10] proposed a new method to calculate channel information between source and relay. A robust beamforming method under imperfect channel knowledge was proposed in [11] to appropriately share spectrum between PUs and secondary users (SUs). A joint scheduling and transmitting scheme for MIMO relay was proposed in [12]. This scheme aimed at ensuring highly spectrum usage to improve the total throughput of the network. Resource allocation problems of MIMO relay in cognitive radio networks were discussed in [13][14][15]. In [13], Li et al. investigated the joint power allocation problem for MIMO-OFDM cognitive radio networks based on environmental learning algorithms. Cooperation of SUs and power allocation were jointly discussed in [14], where a low complexity approach was proposed to solve the resource allocation problem to maximize the achievable data rate of SUs. AbbasiJannatabad et al. in [15] studied the cooperative beamforming and relay selection problem, and power allocation was also considered. In [16], Gao et al. explored the MIMO-empowered CR network to achieve the ultimate flexibility and efficiency in dynamic spectrum access and spectrum utilization. They developed a mathematical model to optimize the maximize throughout. In contrast to the above work, we implement a real system of cooperative MIMO relay that involves VSMC MIMO scheme to demonstrate the high performance of the proposed scheme.

\section{VSMC MIMO SCHEME IN COOPERATIVE MIMO RELAY}

In this section, we describe how to implement cooperative MIMO relay using the VSMC MIMO scheme in cognitive radio networks. First, we give a simple example to illustrate why we select some MIMO (or SISO) nodes as relays in cognitive radio networks. Then, we explain why the VSMC MIMO scheme is suitable to be implemented in MIMO (or SISO) relays, and finally we present the details on how to implement the VSMC MIMO scheme.

\section{A. Cooperative MIMO Relay}

In the network we consider, there are several PUs that have the licensed spectrum, one access point (AP) and several SUs. This AP has multiple antennas to get the spatial multiplexing/diversity gain. Some SUs are equipped with multiple antennas while others with single antenna. The SUs are allowed to use the spectrum of the PUs to communicate with AP when PUs do not occupy the spectrum. The AP and SUs periodically sense the spectrum to update the information of availability of channels.

We explain an example of cooperative MIMO relay in cognitive radio network shown in Fig. 2(a). The AP is equipped with three antennas, and three SUs D1, D2 and $R 1$ are equipped with one, two and three antennas. During 


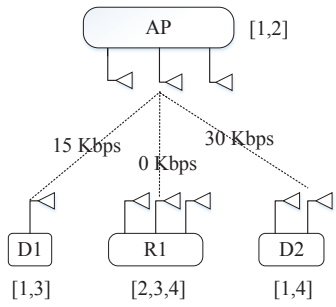
of hybrid nodes

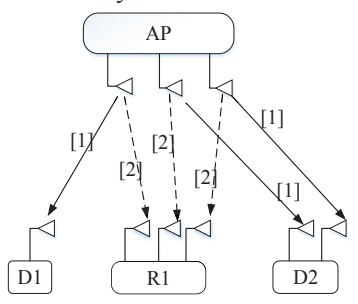

(c) MIMO relay scheme (time slot 1) (a) Channel and demand

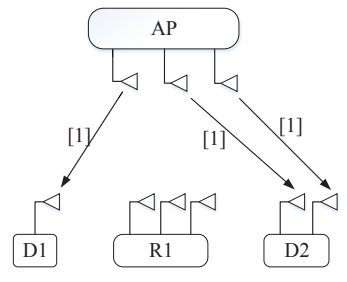

(b) Traditional Approach

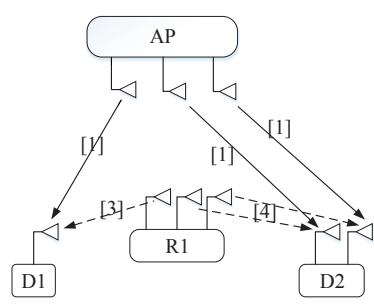

(d) MIMO relay scheme (time slot 2)
Fig. 2. An example for cooperative MIMO relays in cognitive radio networks

a transmission time period, the channel conditions of the four nodes are also shown in Fig. 2(a). Two nodes can communicate with each other if they share common channels. We assume that the data rate of each stream on one channel is $10 \mathrm{Kbps}$. We also assume that MIMO nodes try to obtain their spatial multiplexing gain to achieve larger throughput. The traffic demands of $D 1, D 2$ and $R 1$ are $15 \mathrm{Kbps}, 30 \mathrm{Kbps}$ and 0 Kbps respectively. In the traditional approach, AP transmit three streams to $D 1$ and $D 2$ on their common channel one while no message is sent to $R 1$ as shown in Fig. 2(b). However, in that case, the traffic demands of $D 1$ and $D 2$ are not satisfied. In addition, spectrum resources such as common channel two between $\mathrm{AP}$ and $R 1$, common channel three between $R 1$ and $D 1$ and common channel four between $R 1$ and $D 2$ are not used. Here, $R 1$ is a resource abundant node, so we can let $R 1$ be a relay for $D 1$ and $D 2$ to relieve their traffic demands. The process is shown in Fig. 2(c)(d). We divide the transmission time into two time slots. In time slot one, AP transmits three streams to $D 1$ and $D 2$ on channel one and three streams to $R 1$ on channel two. In time slot two, AP keeps transmitting three streams to $D 1$ and $D 2$ on channel one, meanwhile, $R 1$ decodes the messages sent by AP, forwards one stream to $D 1$ on channel three and two streams to $D 2$ on channel four. In this case, the otherwise wasted channel two, three, four are well used and the traffic demands of $D 1$ and $D 2$ can be satisfied. The total throughput of the network increases $50 \%$, the throughput of $D 1$ and $D 2$ increases $50 \%$ respectively.

\section{B. Utilization of VSMC MIMO scheme}

The VSMC MIMO scheme can be used to implement cooperative MIMO relay in cognitive radio networks. From the above analysis, we conclude that using resource abundant nodes as relays can significantly improve the total throughput of the network. And to implement the MIMO relay, one MIMO node needs to transmit variable numbers of streams on multiple channels to multiple destinations, e.g., the AP in time slot one and $R 1$ in time slot two. The VSMC MIMO scheme can effectively solve this problem and can be implemented on the cooperative MIMO relay.

\section{Fundamental Components of VSMC MIMO scheme}

To implement VSMC MIMO scheme, we have two considerations. First, one radio should transmit or receive signals on multiple (probably discontiguous) channels at the same time. Second, the interferences of several concurrent transmissions should be eliminated.

We adopt the Discontiguous Orthogonal Frequency Division Multiplexing (D-OFDM) scheme [17] to allow a single radio to operate on multiple channels. In D-OFDM scheme, several contiguous orthogonal subcarriers can be combined into a channel, and we can modulate the message onto the corresponding subcarriers to transmit message on multiple channels.

The transmitters and receivers can be viewed as a "virtual MIMO" system on a specific channel, so we can use precoding methods to remove the impact from data streams on the same channel. Most of the pre-coding methods of MIMO system can be used in this VSMC MIMO scheme. Pre-coding methods such as the Space Time Block Code (STBC), the Space Time Trellis Code (STTC) can achieve diversity gain, and the Vertical Bell Laboratories Layered Space-Time (VBLAST) code can obtain spatial multiplexing gain. In this paper, we use V-BLAST in our scheme to obtain spatial multiplexing gain and achieve high data rate.

We combine the D-OFDM scheme with MIMO techniques to implement VSMC MIMO scheme, and the fundamental components of the VSMC MIMO scheme are as follows.

1) Baseband Signal Processing: In the VSMC scheme, receivers should receive signal on some channels while eliminate signal on other channels. To correctly receive and decode signal on multiple channels, we use a low-pass filter to get the desired signal on certain channels in baseband, which can remove noises and interferences from other channels. Assume that the desired baseband signal is on channel one, whose frequency range is $\left[f_{1}, f_{2}\right]$. On the receiver side, the first step is to shift the frequency of the received signal by $\frac{f_{1}+f_{2}}{2}$. Then, the signal pass the corresponding low-pass filter. After that, the signal are shift back by $-\frac{f_{1}+f_{2}}{2}$. By doing this, signal in the symmetric frequency $\left[-f_{2},-f_{1}\right]$ is not kept, and the interferences from other channels are removed.

2) Preambles on Part of Subcarriers: The VSMC MIMO scheme needs to transmit and receive packets on part of the subcarriers that are related to the working channels. As a consequence, the preambles for time synchronization and the training sequences for channel estimation should be added individually on the corresponding subcarriers. Based on the preambles added before each packet, we can use correlation algorithm to detect the beginning of each packet. The preambles are also used to calculate the carrier frequency offset. We assume that the channel is quasi-stationary and does not change during the transmission, so the training sequences on the certain subcarriers can be used to estimate the CSI between transmitters and receivers. 
3) Coding Methods in VSMC MIMO scheme: We use VBLAST pre-coding method in VSMC MIMO scheme. Other pre-coding methods can also be used to in VSMC MIMO system. There are several decoding algorithms for V-BLAST, such as Zero Forcing (ZF), Minimum Mean Square Error (MMSE), and Maximum Likelihood (ML). In our scheme, we adopt $\mathrm{ZF}$ algorithm on the receiver side. ZF algorithm is a low-complexity algorithm which is widely used in wireless system. Under high signal-to-noise-ratio (SNR) and perfect CSI condition, ZF algorithm can effectively achieve the spatial multiplexing gain of MIMO system.

4) Channel States Information: In ZF algorithm, the MIMO receivers have to calculate the CSI between transmitters and receivers. The CSI is estimated by the method Linear Least Squares Estimate (LLSE). We have to calculate the CSI based on the training sequences inserted on the corresponding subcarriers. The CSI is the fundamental component of ZF algorithm in V-BLAST pre-coding method.

\section{Resource Allocation}

In this section, we move on to the network perspective. In practical cognitive radio networks, there are several SUs, that are served by one AP in a certain area. We only consider downlink transmission in our work since the analysis of uplink transmission is similar to downlink. To implement MIMO relays in cognitive radio networks, we need to appropriately allocate the spectrum to nodes and select nodes as relays. We first model the network, then formulate the resource allocation problem and finally propose a heuristic solution to this problem.

\section{A. Network Model}

We consider a cognitive radio network with one AP and $N$ SUs [7]. AP is a MIMO node, and SUs consist of both MIMO nodes and SISO nodes. $\mathcal{V}=\left\{v_{0}, v_{1}, \ldots, v_{N}\right\}$ represents the $N+1$ nodes, where $v_{0}$ denotes the AP node, and $\left\{v_{1}, v_{2}, \ldots, v_{N}\right\}$ are SU nodes. $\mathcal{E}=\left\{e_{i j}\right\}_{(N+1) \times(N+1)}$ represents the direct links between nodes, where $e_{i j}=\{0,1\}$ $(0 \leq i, j \leq N) \cdot e_{i j}=1$ means there exists a direct link between node $v_{i}$ and $v_{j}$, and $e_{i j}=0$ otherwise. We assume that the direct link between two nodes is bidirectional and thus symmetrical, that is, $e_{i j}=e_{j i}$. We use $\mathcal{D}=\left\{d_{1}, d_{2}, \ldots, d_{N}\right\}$ to denote the data demands that $\mathrm{SU}$ nodes need to receive from $v_{0}(\mathrm{AP})$.

We define the antenna matrix $\mathcal{A}=\left\{a_{0}, a_{1}, \ldots, a_{N}\right\}\left(a_{i} \geq\right.$ $1,0 \leq i \leq N)$, which represents the number of antennas that each node is equipped with.

We assume that the available frequency range is $\left[f_{1}, f_{2}\right]$, which is channelized into $K$ channels with same bandwidth, given channel matrix $\mathcal{H}=\left\{\vec{h}_{0}, \vec{h}_{1}, \ldots, \vec{h}_{N}\right\}$, where $\vec{h}_{i}$ is a $K$-dimension vector that represents the channel availability at node $v_{i}$. In some areas of the cognitive radio network, parts of the channels are occupied by PUs, so these channels are blocked for secondary transmission. We ignore the heterogeneity of the available channels at each node, and write $\vec{h}_{i}$ as $\vec{h}_{i}=\left\{h_{i}^{1}, h_{i}^{2}, \ldots, h_{i}^{K}\right\}$, where $h_{i}^{k}=\{0,1\}$
$(1 \leq k \leq K, 0 \leq i \leq N) . h_{i}^{k}=1$ means that the channel $k$ at node $v_{i}$ is available, and $h_{i}^{k}=0$ means unavailable.

\section{B. Problem Formulation}

In this subsection, we formulate the joint channel allocation and relay selection problem. We denote the channel assignment matrix as $\mathcal{C}=\left\{\vec{c}_{i j}\right\}_{(N+1) \times(N+1)}$, where $\vec{c}_{i j}=\left\{c_{i j}^{1}, c_{i j}^{2}, \ldots, c_{i j}^{K}\right\}(0 \leq i, j \leq N)$ and $c_{i j}^{k}=\{0,1\}$ $(1 \leq k \leq K, 0 \leq i, j \leq N), c_{i j}^{k}=c_{j i}^{k} . c_{i j}^{k}=1$ means that channel $k$ is assigned to link $e_{i j}$ for data transmission, and $c_{i j}^{k}=0$ otherwise. Thus, allocating channels to nodes for transmission transforms to properly choosing the value $c_{i j}^{k}$ $(0 \leq i, j \leq N)$. The constraint of channel assignment can be given:

$$
c_{i j}^{k} \leq h_{i}^{k} h_{j}^{k}, 1 \leq i, j \leq N, \forall k .
$$

Constraint (1) indicates that channel $k$ can be assigned to link $e_{i j}$ for transmission if and only if channel $k$ is available at both node $v_{i}$ and $v_{j}$.

Since the VSMC MIMO scheme is adopted in our system, a node can transmit or receive multiple data streams on one channel concurrently. We denote the data streaming matrix as $\mathcal{S}=\left\{\vec{s}_{i j}\right\}_{(N+1) \times(N+1)}$, where $\vec{s}_{i j}=\left\{s_{i j}^{1}, s_{i j}^{2}, \ldots, s_{i j}^{K}\right\}(0 \leq$ $i, j \leq N)$ is a $K$-dimension vector, and $s_{i j}^{k}(1 \leq k \leq K, 0 \leq$ $i, j \leq N$ ) denotes the amount of data streams transmitted on link $e_{i j}$ on channel $k, s_{i j}^{k}=s_{j i}^{k}$. We regard the system as a "virtual MIMO" system, so the constraints for $s_{i j}^{k}(0 \leq i, j \leq$ $N)$ can be given:

$$
\begin{aligned}
& \sum_{j=0}^{N} s_{i j}^{k} \leq \min \left\{a_{i}, \sum_{j=0}^{N} c_{i j}^{k} a_{j}\right\}, \forall i, \forall k, \\
& \sum_{i=0}^{N} s_{i j}^{k} \leq \min \left\{a_{j}, \sum_{i=0}^{N} c_{i j}^{k} a_{i}\right\}, \forall j, \forall k .
\end{aligned}
$$

Constraint (2) (3) indicate that the amount of streams can not exceed the minimum number of antennas of the transmitters and receivers.

We need to properly schedule the concurrent transmissions and choose the relay nodes in the network to maximize the total throughput. Therefore, we define a relay matrix $\mathcal{R}=$ $\left\{r_{i j}\right\}_{N \times N}$, where $r_{i j}=\{0,1\}(1 \leq i, j \leq N)$ represents whether node $v_{i}$ help node $v_{j}$ relay data transmission or not; $r_{i j}=1$ means node $v_{i}$ works as a relay node for node $v_{j}$, and $r_{i j}=0$ otherwise. We assume that one node can be a relay to several nodes and one node can have several relays. Assume this system is half duplex, i.e., the transmission between two nodes is unidirectional at any time. We have the following constraints for relays.

1) channel availability: the relay link can exist if and only if there exist assigned available channels between two nodes, i.e.,

$$
r_{i j} \leq \max \left\{c_{i j}^{1}, c_{i j}^{2}, \ldots, c_{i j}^{K}\right\}, 1 \leq i, j \leq N .
$$


2) half duplex: if a node is relayed by other nodes, it cannot be a relay, i.e.,

$$
r_{i j} \sum_{j^{\prime}=1}^{N} r_{j^{\prime} i}=0,1 \leq i, j, j^{\prime} \leq N .
$$

We denote the data transmission rate matrix as $\Phi=$ $\left\{\vec{\phi}_{i j}\right\}_{(N+1) \times(N+1)}$, where $\vec{\phi}_{i j}=\left\{\phi_{i j}^{1}, \phi_{i j}^{2}, \ldots, \phi_{i j}^{K}\right\}(0 \leq$ $i, j \leq N)$, and $\phi_{i j}^{k}(1 \leq k \leq K, 0 \leq i, j \leq N)$ denotes the data transmission rate of link $e_{i j}$ on channel $k$. We partition time into time frames with fixed length $\Delta t$. Since there can be two kinds of transmission in the network: one is direct transmission, and the other is relay transmission. In the relay transmission process, node $v_{0}$ first transmits data to relay $v_{i}$ in the first half of time in one frame, and then relay $v_{i}$ retransmits the message to end user $v_{j}$ in the next half of the frame. Assume that for any transmission link $e_{i j}$ in any given time period, the transmission rate $\xi$ is constant on any data stream. Thus, given a link $e_{i j}$ and its stream vector $\vec{s}_{i j}$, its data transmission rate for link $e_{i j}$ can be expressed as

$$
\phi_{i j}=\sum_{k=1}^{K} \phi_{i j}^{k}=\sum_{k=1}^{K} \xi s_{i j}^{k} .
$$

Given the transmission rate and transmission time, we can calculate the throughput for SU nodes as $\Gamma=\left\{\gamma_{1}, \gamma_{2}, \ldots, \gamma_{N}\right\}$. We observe that, if node $v_{i}$ receives data from $v_{0}$ (AP) on channel $k$ directly, the average throughput of link $e_{0 i}$ on channel $k$ is

$$
\gamma_{0 i}^{k}=\frac{\phi_{0 i}^{k} \Delta t}{\Delta t}=\phi_{0 i}^{k}
$$

However, if node $v_{i}$ acts as a relay, it receives data from $v_{0}$ on channel $k$, and forwards the data to node $v_{j}$ on channel $k^{\prime}$, then the average throughput of link $e_{0 i}$ on channel $k$ reduces to

$$
\gamma_{0 i}^{k}=\frac{\phi_{0 i}^{k} \Delta t}{2 \Delta t}=\frac{1}{2} \phi_{0 i}^{k},
$$

and the average throughput of link $e_{i j}$ on channel $k^{\prime}$ is the same if the capacity of link $e_{i j}$ is large enough, namely $\gamma_{i j}^{k^{\prime}}=$ $\frac{1}{2} \phi_{0 i}^{k}$, since $v_{i}$ only receives $\frac{1}{2} \phi_{0 i}^{k} \Delta t$ message from $v_{0}$.

Next we analyze the average throughput of nodes in the network. We can divide the nodes into three categories: directreceiving nodes, relay nodes and relay-assisted nodes. We calculate throughputs of these three kinds of nodes individually as follow:

1) direct-receiving node: if all transmissions of node $v_{i}$ are direct transmission and no relays are involved, i.e., $\sum_{j=1}^{N} r_{i j}=0$ and $\sum_{j=1}^{N} r_{j i}=0$, the throughput of node $v_{i}(1 \leq i \leq N)$ is

$$
\left.\gamma_{i}\right|_{d}=\min \left(\sum_{k=1}^{K} \xi s_{0 i}^{k}, d_{i}\right),
$$

2) relay node: if node $v_{i}$ acts as a relay, the throughput of own data of node $v_{i}(1 \leq i \leq N)$ is

$$
\left.\gamma_{i}\right|_{r}=\min \left(\sum_{k=1}^{K} \frac{1}{2} \xi s_{0 i}^{k}, d_{i}\right),
$$

3) relay-assisted node: if node $v_{i}$ receives data from node $v_{0}$ with the help of relay node $v_{j}(1 \leq j \leq N)$, i.e., $r_{j i}=1$, the throughput of node $v_{i}(1 \leq i \leq N)$ is:

$$
\begin{array}{r}
\left.\gamma_{i}\right|_{h}=\min \left(\sum_{k=1}^{K} \phi_{0 i}^{k}+\sum_{j=1}^{N} r_{j i}\right. \\
\left.\min \left(\sum_{k=1}^{K} \frac{1}{2} \phi_{j i}^{k}, \sum_{k=1}^{K} \frac{1}{2} \phi_{0 j}^{k}-\left.\gamma_{j}\right|_{r}\right), d_{i}\right) .
\end{array}
$$

Under a certain relay relationship $\mathcal{R}$ and channel selection $\mathcal{C}$, the throughput of node $v_{i}(1 \leq i \leq N)$ can be written as

$$
\gamma_{i}=\left.(1-R)\left(1-R_{a}\right) \gamma_{i}\right|_{d}+\left.R \gamma_{i}\right|_{r}+\left.R_{a} \gamma_{i}\right|_{h},
$$

where

$$
\begin{gathered}
R=\left\{\begin{array}{l}
1, \text { if } \sum_{j=1}^{N} r_{i j} \geq 1 . \\
0, \text { otherwise } .
\end{array}\right. \\
R_{a}=\left\{\begin{array}{l}
1, \text { if } \sum_{j=1}^{N} r_{j i} \geq 1 . \\
0, \text { otherwise } .
\end{array}\right.
\end{gathered}
$$

Then the optimization problem is, given demands of each node $\mathcal{D}$, to find a feasible relay selection and channel allocation strategy $\{\mathcal{R}, \mathcal{C}\}$ such that the total throughput of the system is maximized. This problem can be expressed as

$$
\begin{aligned}
& \max _{\{\mathcal{D}, \mathcal{R}, \mathcal{C}\}} \sum_{i=1}^{N} \gamma_{i}, \\
& \text { s.t. }(1)(2)(3)(4)(5) .
\end{aligned}
$$

\section{Heuristic Solution for Resource Allocation}

The optimization problem (15) is complex, and it is difficult to obtain the close-form solution. Thus, we propose a heuristic solution in centralized manner to solve this problem. First, we propose the following proposition.

Proposition 1 (Node selfishness): Assume that all users (nodes) are individual rational, and if the traffic demand of a node is not satisfied, it will not act as a relay for other nodes. It is easy to understand and we omit the proof here.

(I) Direct transmission: We need to obtain achievable data rates of nodes and compare achievable data rates with their traffic demands. Therefore, we try to achieve the maximum throughput of the network without relay transmission, the optimization problem (15) can be simplified to

$$
\begin{aligned}
& \max _{\{\mathcal{D}, \mathcal{H}\}} \sum_{i=1}^{N} \min \left(\sum_{k=1}^{K} \xi s_{0 i}^{k}, d_{i}\right), \\
& \text { s.t. }(1)(2)(3) .
\end{aligned}
$$

The joint channel selection and channel assignment problem for direct transmission is similar to that of [7]. And this problem can be transformed to a max flow problem. 
Given $\mathcal{D}$ and $\mathcal{H}$, we can draw a graph $G_{1}$ representing the direct transmission procedures. We regard the AP as the source node. Each channel is denoted by a channel node $k(1 \leq k \leq$ $K$ ). If channel $k$ is available at $\mathrm{AP}$, the channel node $k$ is connected to source node with the weight $a_{0} \xi$. Every SU is represented by a user node. If channel $k$ is available at node $v_{j}$, the corresponding node is connected to channel node $k$ with weight $a_{j} \xi$. All the SUs are connected to a sink node $X_{1}$, and the weight of each link is the corresponding user demand in $\mathcal{D}=\left\{d_{1}, d_{2}, \ldots, d_{N}\right\}$. After that, the optimization problem (16) is transformed to a max-flow problem, and we can use Hungarian Algorithm to solve the channel assignment problem in polynomial time.

After solving the max-flow problem, we mark $c_{0 j}^{k}=1$ and let $s_{0 j}^{k}$ equal to the weight of the path divided by $\xi$ if the path between channel node $k$ and user node $v_{j}$ is chosen, otherwise $c_{0 j}^{k}=s_{0 j}^{k}=0$. Thus, we have the results of channel assignment and data stream assignment between the AP and the user nodes. Next, we can know whether user node $v_{i}$ is self-sufficient or not, based on whether its data demand $d_{i}$ is less than or equal to the direct transmission throughput $\left.\gamma_{i}\right|_{T H}$ or not, where $\left.\gamma_{i}\right|_{T H}=\sum_{k=1}^{K} \xi s_{0 i}^{k}$. If $d_{i}<\left.\gamma_{i}\right|_{T H}$, the node $v_{i}$ can fulfill its data demand with excessive ability to transmit data, so it is a potential relay of other nodes and we add it to set $Y$; otherwise, if $d_{i}>\left.\gamma_{i}\right|_{T H}$, the node cannot support itself and needs help from relay(s), then we add it to set $Z$. Thus we can get two sets of nodes, and the next step is to match the nodes in these two sets.

(II) Relay selection: In this step, we find a match $M$ that maximize the overall throughput of the network. That is, for each node in $Z$, we find its matching node(s) in $Y$ to serve as relay(s), and allocate the corresponding channel(s), so that (15) can be solved.

This is a complex matching problem. First, for each node in $Y$, we calculate its relay potential $\mathcal{P}=\left\{p_{i} \mid v_{i} \in Y\right\}$, where $p_{i}=\frac{1}{2}\left(\sum_{k=1}^{K} \xi s_{0 i}^{k}\right)-d_{i}$. If $p_{i} \leq 0, v_{i}$ is removed from $Y$, and for each node in $Z$, we calculate its data gap $\mathcal{G}=$ $\left\{g_{j} \mid v_{j} \in Z\right\}$, where $g_{j}=d_{j}-\sum_{k=1}^{K} \xi s_{0 j}^{k}$.

Once $\mathcal{P}$ and $\mathcal{G}$ are obtained, we can create graph $G_{2}$. We first set a source node $S$. Each node $v_{i}$ in set $Y$ is denoted by node $R_{i}$ in $G_{2}$. There are links between $S$ and every $R_{i}$, and the weight of each link is $p_{i}$. Channel $k$ that satisfies $c_{0 i}^{k}=0$ is denoted by a channel node $C_{k}$, channels that do not satisfy it are not included in graph $G_{2}$ since those channels are occupied by the direct communication with AP. Each node $v_{j}$ in set $Z$ is represented by a destination node $D_{j}$. If channel $k$ is available at node $v_{i}$, there is a link between $R_{i}$ and $C_{k}$ with the weight $\frac{1}{2} a_{i} \xi$. If channel $k$ is available at node $v_{j}$, there is a link between $D_{j}$ and $C_{k}$ with the weight $\frac{1}{2} a_{j} \xi$. All $D_{j}$ connect to sink node $X_{2}$ with their corresponding weight $g_{j}$. Thus, this problem is also a max-flow problem, and we can solve it using Hungarian Algorithm, or other greedy algorithm in polynomial time.

After solving the second max-flow problem, we mark $r_{i j}=$ 1 if the path between node $v_{i}$ and user node $v_{j}$ is chosen,

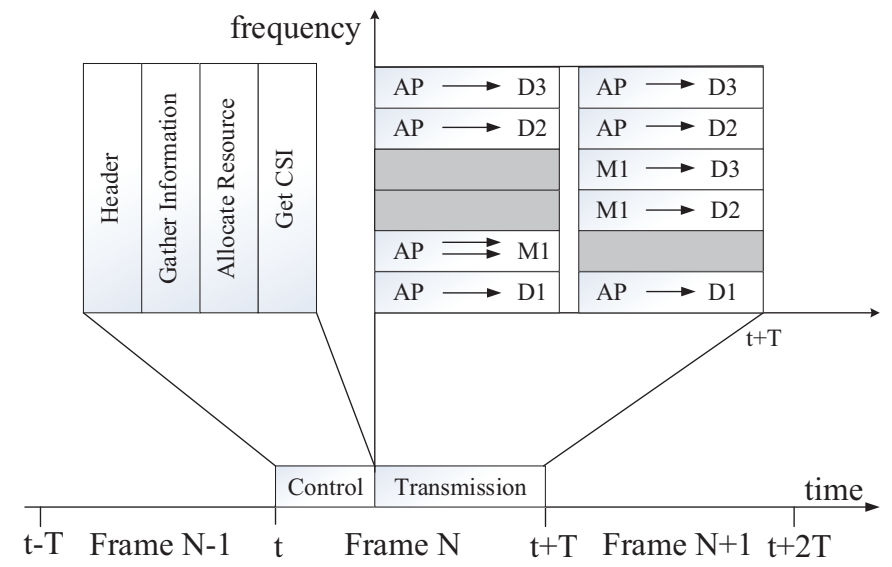

Fig. 3. MAC layer design

otherwise $r_{i j}=0$. Thus, we obtained the relay assignment $\mathcal{R}$. With the results of these two steps, we can get the complete channel assignment $\mathcal{C}$ and data stream assignment $\mathcal{S}$, so the whole relay selection and resource allocation are completed.

\section{MAC LAYER DESIGN}

The analysis in previous sections demonstrates that using VSMC MIMO scheme to implement cooperative MIMO relay in cognitive radio networks can significantly improve the total throughput of the network. However, implementing cooperative MIMO relay needs good organization and coordination of all the nodes in the network. To make cooperative MIMO relay scheme workable and reliable, we propose a MAC layer protocol to coordinate all the nodes in the network.

We assume that the whole network is in global synchronization [7]. The transmission process is based on frames, and the length of the frame is fixed and properly set to avoid intolerant disturbance to the PUs. The frame structure is depicted in Fig. 3. Each frame is composed of two parts: control part and transmission part. The control part is for sharing control message, and the transmission part is for transmitting message. We assume that there is a common channel among all the nodes in the network, and nodes share information on this common channel during the time period of control part. Then during the time period of the transmission part, nodes turn to their assigned channels to transmit message.

In our scheme, AP acts as a central controller. In the control part, first, AP broadcasts a header to all the nodes in the network to synchronize all the nodes. Then, nodes gather the information of their traffic demands and available channels and send the information to AP in order. Next, AP uses the gathered information to run the relay selection and channel allocation algorithm. The result of resource allocation is broadcast on the common channel to all nodes. After that, if pre-coding methods need information of CSI on the transmitter side, nodes can transmit training sequences on their assigned channels to AP and then relay-assisted nodes transmit training sequences to their relay nodes to calculate the corresponding CSI. Otherwise, the control part is over.

In the transmission part, nodes start to communicate with each other based on the resource allocation decision made 


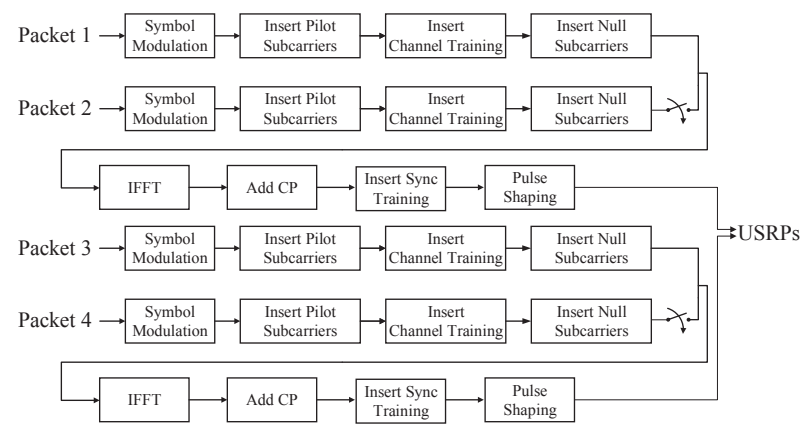

Fig. 4. Transmitting chain

by AP. If a node directly communicate with AP, it receives message from AP on the assigned channels during the transmission part, e.g. $D 1$ in Fig. 3. If a node is selected as a relay, e.g. $M 1$, first, it receives message from AP during the first half of time in transmission part, then retransmits message to its destinations, e.g. $D 2$ and $D 3$, in the next half of time in transmission part. Nodes such as $D 2$ and $D 3$ receive message from AP during the first half of time in transmission part and then receive message from AP and their relays in the second half of time in transmission part. There is a small time interval between these two transmission parts, which is important for relay nodes to switch work mode, decode and precode messages, and synchronize with other nodes.

\section{TeStbed Description AND EXPERIMENTAL RESULTS}

In this section, we first describe the system of cooperative MIMO relay in cognitive radio networks. Then, we present the experiments and evaluate the experimental results.

\section{A. Testbed Description}

We implement the system of cooperative MIMO relay that involves VSMC MIMO scheme in cognitive radio networks based on USRP and LabVIEW. We implement VSMC MIMO scheme based on the existing codes of MIMO technology introduced in [18]. Our system includes a secondary MIMO AP and several secondary SISO and MIMO end users. Each node consists of one or multiple USRPs. The nodes are connected to a general purpose computer through a Gigabit Ethernet Switch. The VSMC MIMO scheme and resource allocation algorithm are implemented in software.

We use the NI USRP 2920, which is designed to operate in the $2.4 \mathrm{GHz}$ or $5 \mathrm{GHz}$ frequency range. The channel bandwidth can reach up to $20 \mathrm{MHz}$, and the maximum gain for transmission is $30 \mathrm{~dB}$. We main process the baseband signal at the NI USRP joint with LabVIEW platform. We use MIMO cables to connect two USRPs as a pair to ensure that they share the same operating frequency. An external clock provides a 10 $\mathrm{MHz}$ clock and Pulse Per Second (PPS) signal (0-5 V, $1 \mathrm{~Hz}$ square-wave) to all the pairs to synchronize all the nodes of the testbed. We use a computer equipped with dual-core 3.20 $\mathrm{GHz}$ processors and $4 \mathrm{~GB}$ of memory to control USRPs.

We implement the system setup in LabVIEW on the PCs. LabVIEW is a graphical programming language that is used

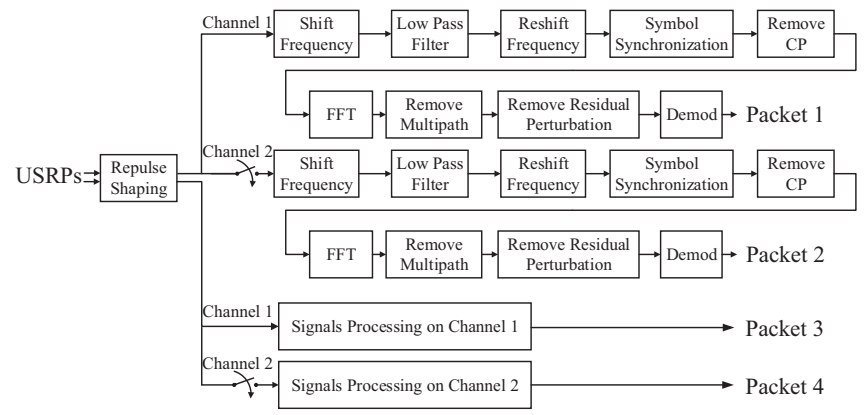

Fig. 5. Receiving chain

to quickly develop systems by controlling hardware with blocks of LabVIEW code called virtual instruments [19]. The processes of transmitting and receiving are shown in Fig. 4. and Fig. 5.

On the transmitter side, each packet is sent through one stream on a specific channel. For a certain channel, we generate the streams using V-BLAST pre-coding method. For each stream, pilot subcarriers are first inserted to help exactly calculate the CSI of the transmitting stream to remove residual perturbation of the signals on the receiver side. Next, training sequences are inserted into multiple packets on corresponding subcarriers. Training sequences for multiple packets are different. Null subcarriers are inserted to guarantee the guard bands between channels. After doing Inverse Fast Fourier Transform (IFFT) and adding cyclic prefix (CP), the preambles for symbol synchronization are added. The symbols then pass a pulse shaping filter and are sent by USRPs.

On the receiver side, receivers open or close switch to get the signals on the desired channels. For each channel, receivers process the corresponding streams. For each stream, the signals first pass a matched filter, then the central frequencies of the expected signals on the channel are shifted to zero. Next, the signals are filtered by the corresponding low-pass filter, and shifted back to the original frequencies. We employ the low-pass filter because its performance is better than that of the band-pass filter or the high-pass filter in LabVIEW. Next, we do the correlation for symbol synchronization, remove $\mathrm{CP}$, and do the Fast Fourier Transform (FFT). After that, we calculate the CSI of all the streams on the channel. With the CSI, we use decode method ZF to get the original signals. After demodulation, we can get the message sent by transmitters.

Some of the modules such as symbols modulation and demodulation in transmitter and receiver chain are implemented natively in LabVIEW, other modules such as correlation for time synchronization and channel estimation are implemented in MATLAB and ported over to LabVIEW using embedded MathScript blocks [19].

There are many latencies in the platform of NI USRPs and LabVIEW, which hamper the performance of the system. For example, the time spent on running MathScript functions is highly relied on the performance of the connected computers. In addition, there is a hardware constraint that USRPs require a continuous stream of data for transmission or else an underflow occurs at the transmitter. We need to pad zeros between packets to guarantee multiple packet transmission, 


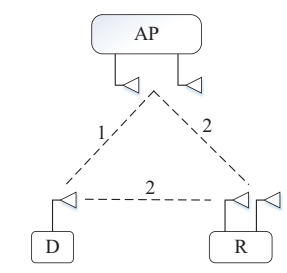

(a) Topology with channels (setup 1)

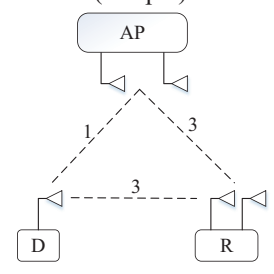

(c) Topology with channels (setup 2)

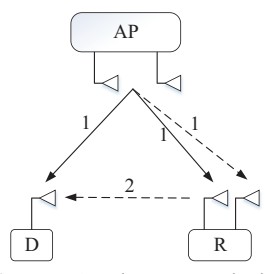

(b) MIMO relay transmissions (setup 1)

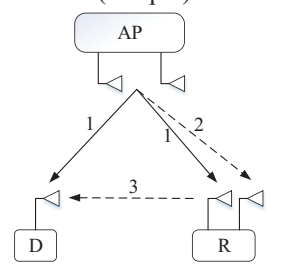

(d) MIMO relay transmissions (setup 2)
Fig. 6. Experiment of two end users

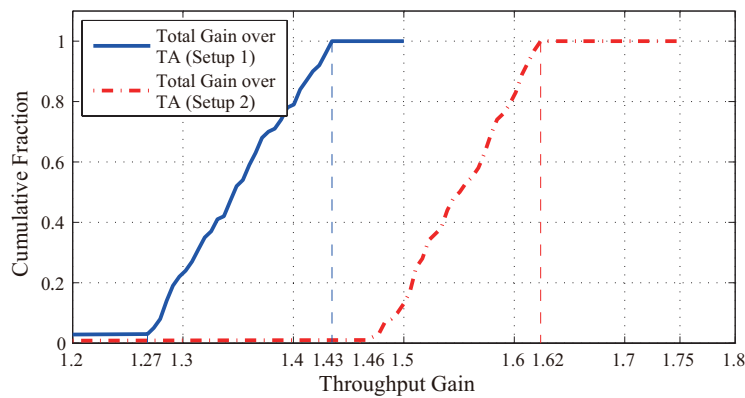

Fig. 7. Total gain of two end users

which lowers the sampling rate of packet transmission. The latencies and constraint have been discussed in [18], We have taken consideration of these latencies and constraint in the experiments.

In the experiments, we compare the results of the cooperative MIMO relay scheme and the traditional approach (TA) without MIMO relay. In the experiments, the I/Q rate is $400 \mathrm{~K} /$ second, the carrier frequency is $915 \mathrm{MHz}$, and pulse shaping samples per symbol is 8 . Each channel is composed of 20 subcarriers, in which 2 subcarriers are used for pilot subcarriers to better calculate the CSI. We set 12 guard subcarriers between two adjacent channels. Each stream can support about $10 \mathrm{Kbps}$ data rate. The actual transmission date varies due to the condition of channels. The data rate is not very large due to the fact that the signals are processed on computers, but the scheme can support larger data rate. Each packet is composed of 2880 bits. Each run transmission 20 packets in downlink. We repeat the downlink transmission for 100 times. We get the throughput of the system in cooperative MIMO relay scheme and traditional approach.

\section{B. Experiments of Two End Users}

First, we design two setups of two end users in a cognitive radio network. The topology of the first setup is shown in Fig. 6(a). In this setup, the AP and node $R$ have two antennas, the other receiver $D$ has only one antenna. The numbers on the dashed lines indicate the numbers of common available

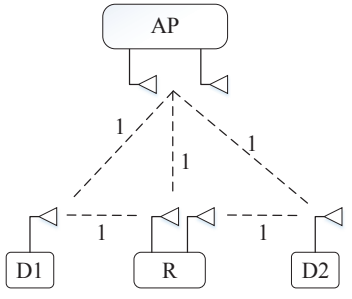

(a) Topology with channels

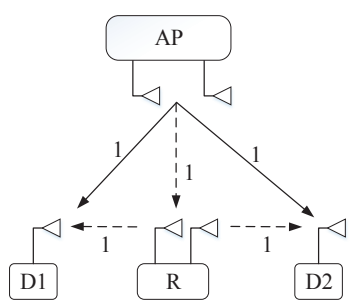

(b) MIMO relay transmissions
Fig. 8. Experiment of three end users

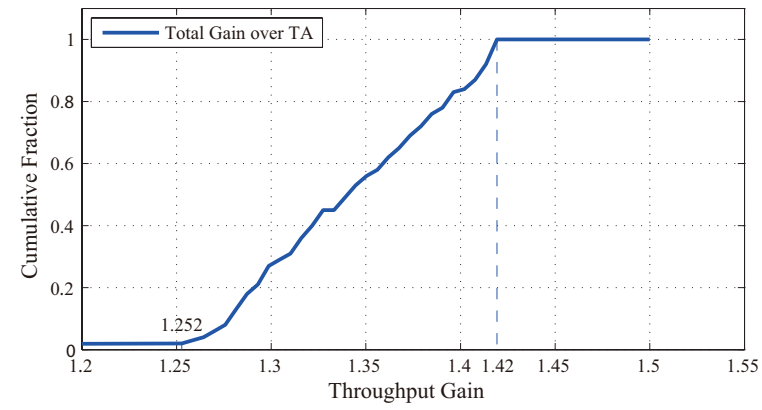

Fig. 9. Total gain of three end users.

channels between two nodes. The traffic demands for $D$ and $R$ are about $25 \mathrm{Kbps}$ and $10 \mathrm{Kbps}$ respectively. In the traditional approach, receiver $R$ could meet its traffic demand but waste large parts of its available spectrum. Receiver $D$, however, could not meet its traffic demand. In the MIMO relay scheme, according to the resource allocation algorithm, relay $R$ can receive four streams on the two available channels during the first time slot and retransmit packets to $D$ in the next time slot, which can improve the throughput of $D$ and the system. The MIMO relay transmission process is described in Fig. 6(b). The solid arrows denote the numbers of transmission channels without MIMO relay and the dotted arrows denote the added working channels in MIMO relay scheme. The gain of the throughput of the system can achieve $50 \%$ in theory by adopting a MIMO relay.

In the next setup shown in Fig. 6(c), the numbers of common available channels between $\mathrm{AP}$ and $R, R$ and $D$ have changed. Other parameters are the same as that of the first setup. According to our resource allocation algorithm, relay $R$ receives five streams during the first time slot, then forwards three streams to $D$ in the next time slot. Node $D$ receives message from AP during all the transmission time. The MIMO relay transmission is shown in Fig. 6(d). The gain of the throughput of the system can achieve $75 \%$ in theory because this one employs more spectrum resources.

The experimental results of two secondary end users are shown in Fig. 7. In the first setup, the average throughput of the system increases $34.74 \%$, compared to the traditional approach without MIMO relays. In the second setup, the average gain of the total throughput of the system achieves $55.02 \%$.

\section{Experiment of Three End Users}

We consider the scenario of three end users in the cognitive radio network. The topology of three end users is shown in Fig. 
8(a). In this case, the AP and receiver $R$ have two antennas as in the previous experiment. The node $D 1$ and $D 2$ have one antenna respectively. The traffic demands for $D 1, D 2$ and $R$ are $15 \mathrm{Kbps}, 15 \mathrm{Kbps}$ and $0 \mathrm{Kbps}$ respectively. According to our algorithm, node $R$ receives packets of $D 1$ and $D 2$, and then forwards them to $D 1$ and $D 2$ as shown in Fig. 8(b). The theoretical gain of the system is $50 \%$.

Our experiment result of three secondary end users is shown in Fig. 9. The total gain of the system is increased by $34.11 \%$.

\section{Evaluation of Experimental Results}

There is a gap between the theoretical analysis and the experimental results. The main reason is that the relay nodes need time to switch from receiving mode to transmitting mode. Also, with the decode-and-forward scheme, relay node has to spend time on decoding and recoding message. Moreover, signal processing rate of the USRP joint with LabVIEW platform is slow and variable, which effects data transmissions.

Comparing the results of the two setups in experiment of two end users, we know that using more spectrum resource would increase the throughput of the network. These results prove the effectiveness of the proposed resource allocation and relay selection algorithm, since the algorithm encourages nodes to utilize the available spectrum as much as possible.

\section{CONCLUSION}

In this paper, we propose a new scheme called VSMC MIMO to make full use of the discontiguous spectrum in cognitive radio networks. VSMC MIMO allows MIMO nodes to transmit variable numbers of streams on multiple channels (which are possibly discontinuous) concurrently. To apply this scheme to implement cooperative MIMO relays in cognitive radio networks, we propose a joint relay selection and channel allocation algorithm, which can maximize the network throughput, and design a corresponding MAC layer protocol to coordinate the nodes in the network. We also build a testbed based on USRP and LabVIEW platforms to implement the proposed scheme in practical cognitive radio networks. The experimental results show that VSMC MIMO can efficiently utilize the discontiguous spectrum and significantly improve the network throughput.

In our future work, we will enlarge the scale of the testbed to evaluate the performance of VSMC MIMO in large-scale MIMO relay systems. Accordingly, we will modify the MAC protocol to an adaptive protocol that is applicable to largescale networks. In addition, we will adopt other precoding methods in VSMC MIMO and evaluate their performances by both analytical and experimental results. Moreover, we will reconstruct the testbed using other software radio platforms, e.g.,WARP [20] and Sora [21].

\section{ACKNOWLEDGMENT}

This work is supported by NSF China (No.61325012, 61271219, 61221001, 61202373, 61102052, 61102051, 61428205, U1405251); China Ministry of Education Doctor Program (No.20130073110025); National Mobile Communications Research Laboratory, Southeast University
(No.2012D13, 2014D07); Shanghai Basic Research Key Project (12JC1405200, 11JC1405100); Shanghai International Cooperation Project (No.13510711300); National Basic Research 973 Program of China (No.2015CB352403); Jiangsu Future Network Research Project (No.BY2013095-1-10).

\section{REFERENCES}

[1] S. Haykin, "Cognitive radio: brain-empowered wireless communications," IEEE J. Sel. Areas Commun., vol. 23, no. 2, pp. 201-220, Feburary 2005.

[2] Q. Liang, X. Wang, X. Tian, and Q. Zhang, "Route-switching games in cognitive radio networks," in Proc. ACM MobiHoc'13, August 2013, pp. 249-252.

[3] J. Mitola, "Cognitive radio — an integrated agent architecture for software defined radio," Ph.D. dissertation, Royal Institute of Technology (KTH), May 2000.

[4] L. Duan, A. W. Min, J. Huang, and K. G. shin, "Attack prevention for collaborative spectrum sensing in cognitive radio networks," IEEE $J$. Sel. Areas Commun., vol. 30, no. 9, pp. 1658-1665, October 2012.

[5] G. Choi, W. Zhang, and X. Ma, "Achieving joint diversity in decodeand-forward MIMO relay networks with zero-forcing equalizers," IEEE Trans. Comm., vol. 60, no. 6, pp. 1545-1554, June 2012.

[6] A. Bansal, M. Bhatnagar, A. Hjorungnes, and Z. Han, "Low-complexity decoding in DF MIMO relaying system," IEEE Trans. Veh. Tech., vol. 62, no. 3, pp. 1123-1137, March 2013.

[7] J. Jia, J. Zhang, and Q. Zhang, "Cooperative relay for cognitive radio networks," in Proc. IEEE INFOCOM, April 2009, pp. 2304-2312.

[8] J. Zhang, J. Jia, Q. Zhang, and E. Lo, "Implementation and evaluation of cooperative communication schemes in software-defined radio testbed," in Proc. IEEE INFOCOM, March 2010, pp. 1-9.

[9] R. Jia, J. Zhang, X. Wang, X. Tian, and Q. Zhang, "Scaling laws for heterogeneous cognitive radio networks with cooperative secondary users," in Proc. IEEE INFOCOM, April 2014, pp. 871-879.

[10] D. Qin, Z. Ding, and S. DasGupta, "On forward channel estimation for mimo precoding in cooperative relay wireless transmission systems," IEEE Trans. Sig. Proc., vol. 62, no. 5, pp. 1265-1278, March 2014.

[11] T. Luan, F. Gao, X. Zhang, J. Li, and M. Lei, "Robust beamforming for relay-aided multiuser MIMO cognitive radio networks," in Proc. IEEE Wireless Communications and Networking Conference (WCNC), April 2013, pp. 2473-2477.

[12] S. Shao, Q. Meng, W. Zong, and W.-P. Zhu, "Relay-assisted MIMO transmission with three-phase scheduling in cognitive radio networks," in Proc. IEEE Wireless Internet Conference (WICON), March 2010, pp. $1-6$.

[13] S. Li, B. Li, C. Xing, Z. Fei, and S. Ma, "Joint resource allocation for learning-based cognitive radio networks with MIMO-OFDM relay-aided transmissions," in Proc. IEEE Wireless Communications and Networking Conference (WCNC), April 2013, pp. 3271-3276.

[14] M. Adian and H. Aghaeinia, "An adaptive cooperative strategy for underlay MIMO cognitive radio networks," in Proc. IEEE ICEE'13, May 2013, pp. 1-6.

[15] M. Abbasi-Jannatabad and H. Khoshbin, "Cooperative beamforming, power allocation and relay selection in MIMO cognitive radio systems," in Sixth International Symposium on Telecommunications (IST), November 2012, pp. 399-403.

[16] C. Gao, Y. Shi, Y. T. Hou, and S. Kompella, "On the throughput of mimo-empowered multihop cognitive radio networks," Mobile Computing, IEEE Transactions on, vol. 10, no. 11, pp. 1505-1519, November 2011.

[17] J. Poston and W. Horne, "Discontiguous OFDM considerations for dynamic spectrum access in idle TV channels," in Proc. IEEE DySPAN'05, November 2005, pp. 607-610.

[18] J. Massey, J. Starr, S. Lee, D. Lee, A. Gerstlauer, and R. Heath, "Implementation of a real-time wireless interference alignment network," in Proc.IEEE Forty Sixth Asilomar Conference on Signals, Systems and Computers (ASILOMAR), November 2012, pp. 104-108.

[19] National Instruments. [Online]. Available: http://www.ni.com/labview/

[20] Rice University, "Warp project." [Online]. Available: http://www.warpproject.org/trac

[21] K. Tan, H. Liu, J. Zhang, Y. Zhang, J. Fang, and G. M. Voelker, "Sora: High-performance software radio using general-purpose multicore processors," Commun. ACM, vol. 54, no. 1, pp. 99-107, Jan. 2011. 